\title{
Computational and experimental methods for the on-line measurement of the apparent viscosity of a crystal suspension
}

C. Herman ${ }^{1}$, F. Debaste ${ }^{1}$, V. Halloin ${ }^{1}$, T. Leyssens ${ }^{2}$, A. Line $^{3} \&$ B. Haut ${ }^{1}$

${ }^{I}$ Transferts, Interfaces and Processes (TIPs), Chemical Engineering Unit, Université Libre de Bruxelles, Bruxelles, Belgium

${ }^{2}$ Automation and Process Control Laboratory (APC),

UCB Pharma, Braine l'Alleud, Belgium

${ }^{3}$ Laboratoire de l'Ingénierie des Systèmes Biologiques et des Procédés (LISBP), Institut National des Sciences Appliquées (INSA) de Toulouse, Toulouse, France

\begin{abstract}
This paper proposes an experimental method, based on the Metzner and Otto concept, for the on-line measurement of the apparent viscosity of a crystal slurry during a crystallization process. The first step of this procedure consists of the determination of the $\mathrm{N}_{\mathrm{p}}-\mathrm{Re}-\mathrm{Fr}$ relation for Newtonian liquids, for two impellertank configurations, chosen such that this relation is a bijective one. This is achieved both experimentally and numerically, using Computational Fluid Dynamics. In the second step of the procedure, the same impeller-tank configurations are used for the determination of the evolution of the apparent viscosity of the crystal slurry (non-Newtonian liquid) during a reference pharmaceutical crystallization process (Etiracetam - UCB). The paper concludes on the influence of the Particle Size Distribution of the crystals on the apparent viscosity of the suspension. For a given crystal mass fraction, the bigger the crystals are - and then the less abundant they are - and the smaller the span is, the smaller the apparent viscosity of the suspension is.
\end{abstract}

Keywords: apparent viscosity, non-Newtonian liquid, suspension, on-line measurement, Computational Fluid Dynamics, crystallization process, Metzner and Otto concept, process rheometer. 


\section{Introduction}

The follow up of a crystallization process can be achieved using several probes. These probes often give information about the Particle Size Distribution (PSD) and form, or the solution concentration, but do not allow estimating any physical characteristics of the suspension such as its apparent viscosity. This physicochemical parameter can be used to characterize and control a crystallization process. Very few on-line experimental techniques exist to directly determine the evolution of the apparent viscosity of a crystal slurry during a crystallization process. Moreover, very few theoretical or empirical correlations of the apparent viscosity of a suspension can be found in the literature. Actually, the correlations proposed are based on the crystal volume fraction [1]. More recent studies show the influence of the PSD on the apparent viscosity of a suspension $[2,3]$.

Therefore, this paper proposes an experimental method to follow the apparent viscosity of a crystal suspension during a crystallization process, based on the Metzner and Otto [4] concept. Using an agitation system as a process rheometer, the method consists of determining the apparent viscosity of a non-Newtonian liquid, as equal to the one of a Newtonian liquid whose agitation, in the same impeller-tank configuration and operational condition, leads to an equal dissipated power.

As presented in Fig.1, the link between the dissipated power, P, and the apparent viscosity, $\mu$, consists of a relation between three dimensionless numbers, the Reynolds number, Re, the Froude number, Fr, and the Power number, $\mathrm{N}_{\mathrm{p}}$, which are respectively defined as follows:

$$
\begin{gathered}
\mathrm{Re}=\frac{\rho N d^{2}}{\mu} \\
F r=\frac{N^{2} d}{g} \\
N_{p}=\frac{P}{\rho d^{5} N^{3}}
\end{gathered}
$$

where $\rho$ is the density of the suspension, $d$ the diameter of the impeller, $N$ the rotational speed of the impeller and $g$ the gravitational acceleration.

For a given impeller-tank configuration, the first step of this procedure consists of the determination of the $N_{p}-R e-F r$ relation for Newtonian liquids. It is achieved using several reference Newtonian liquids of known density, $\rho$, and viscosity, $\mu$. The determination of the power dissipated in the liquid when it is agitated with an impeller of diameter $\mathrm{d}$, at a fixed rotational speed, N, allows determining the three dimensionless numbers (Fig.1). These determinations are realized experimentally and numerically, using Computational Fluid Dynamics (CFD). The relation between $\mathrm{Re}, \mathrm{Fr}$ and $\mathrm{N}_{\mathrm{p}}$, in the laminar and intermediate Reynolds range, is proposed such as $[1,5]$ :

$$
N_{p}=c \operatorname{Re}^{x} F r^{y}
$$


The unknown parameters, $\mathrm{c}, \mathrm{x}$ and $\mathrm{y}$, which depend on the impeller-tank configuration, are identified by a least square optimisation. In the turbulent Reynolds range, the Power number is constant (independent on Re and Fr).

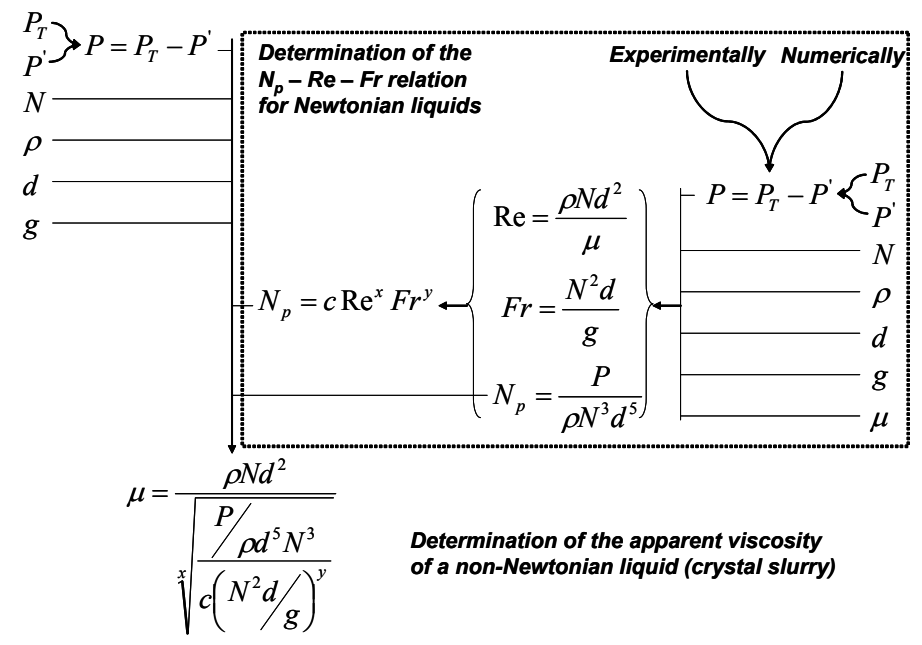

Figure 1: Schematical presentation of method to determine the apparent viscosity of a suspension, based on the Metzner and Otto [4] concept.

In the second step of the procedure, the same impeller-tank configuration is used for the determination of the apparent viscosity of a non-Newtonian liquid. For this purpose, the power dissipated in the liquid is measured when it is agitated with the impeller of diameter $d$, rotating at a fixed rotational speed, $\mathrm{N}$. Knowing the density, $\rho$, of the suspension, the inversion of the $N_{p}-\operatorname{Re}-\mathrm{Fr}$ relation allows determining its apparent viscosity. This method can only be used in the Reynolds range in which the $\mathrm{N}_{\mathrm{p}}-\mathrm{Re}-\mathrm{Fr}$ relation is a bijective one.

$$
\mu=\frac{\rho N d^{2}}{\sqrt[x]{\frac{P}{c\left(N d^{5} N^{3} / g\right)^{y}}}}
$$

The method is then applied in the second part of the paper for the determination of the evolution of the apparent viscosity of the crystal slurry during the crystallization process of a reference pharmaceutical compound. 


\section{Materials and methods}

\subsection{Introduction}

The experimental device consists of an unbaffled double-jacketed glass tank of 11 (Fig.2). The mixing of the liquid is ensured by a stirrer equipped with two impellers types: an Anchor or a 4-arms Flat Blade Turbine. The characteristics about the two impeller-tank configurations are reported in Tab.1.

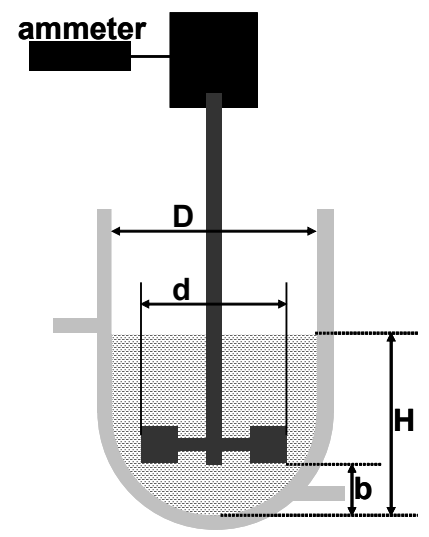

Figure 2: Schematical presentation of the experimental installation.

Table 1: Impeller-tank configurations.

\begin{tabular}{lll}
\hline Impeller & Anchor & 4-arms Flat Blade Turbine \\
\hline & & \\
& & \\
& & \\
\hline $\mathrm{D}(\mathrm{cm})$ & 10 & 10 \\
$\mathrm{H}(\mathrm{cm})$ & 10 & 10 \\
$\mathrm{~d}(\mathrm{~cm})$ & 8.9 & 3.9 \\
$\mathrm{~b}(\mathrm{~cm})$ & 2.5 & 3.1 \\
\hline
\end{tabular}

As the two impeller-tank configurations used are not standard ones, very few results about the $N_{p}-R e-F r$ relation of these systems can be found in the literature. Therefore, this relation (Eq.(4)) is here determined, both experimentally and numerically, for each of the two impeller-tank configurations used. 


\subsection{Experimental determination of the $N_{p}-R e-F r$ relation for Newtonian liquids}

In order to determine this relation for the two impeller-tank configurations in an extended range of Reynolds number (Re), different Newtonian liquids are used:

1. In order to investigate the low Re range, a mixture of glycerine and water $(97 \%)$ at different temperatures $\left(10^{\circ} \mathrm{C}, 15^{\circ} \mathrm{C}, 20^{\circ} \mathrm{C}, 25^{\circ} \mathrm{C}\right.$, $30^{\circ} \mathrm{C}, 35^{\circ} \mathrm{C}, 40^{\circ} \mathrm{C}, 50^{\circ} \mathrm{C}$ and $\left.60^{\circ} \mathrm{C}\right)$ is used. Several rotational speeds (100 rpm, $200 \mathrm{rpm}, 300 \mathrm{rpm}, 400 \mathrm{rpm}$ and $500 \mathrm{rpm})$ are used. The density and the viscosity of the mixture are determined experimentally with a falling sphere viscosimeter (Tab.2).

2. A mixture of salt and water (300 $\mathrm{g}$ of salt per $\mathrm{kg}$ of water) at different temperatures $\left(5^{\circ} \mathrm{C}, 10^{\circ} \mathrm{C}, 15^{\circ} \mathrm{C}\right.$ and $\left.20^{\circ} \mathrm{C}\right)$ is used to investigate the intermediate Re range. $300 \mathrm{rpm}$ is used as the rotational speed of the impeller device. The density and the viscosity of the mixture are provided by Solvay S.A. (Tab.2).

3. Information for the high Re range is obtained using the methanol solvent at ambient temperature $\left(20^{\circ} \mathrm{C}\right)$. The rotational speeds used extend between $100 \mathrm{rpm}$ and $450 \mathrm{rpm}$ by $50 \mathrm{rpm}$ steps. The density and the viscosity of methanol are obtained from literature (Tab.2).

For each experiment, the tank is filled with $10 \mathrm{~cm}$ of the reference Newtonian liquid. The power dissipated in the liquid is determined using an on-line ammeter (APPA 350) (Fig.2). The dissipated power is obtained deducing the value measured at no load ( $\left.\mathrm{P}^{\prime}\right)$ from the observed total power $\left(\mathrm{P}_{\mathrm{T}}\right)$ value (Fig.1).

\subsection{Numerically determination of the $N_{p}-R e-F r$ relation for Newtonian liquid in the turbulent range}

The CFD simulations are realized with the commercial codes Gambit 2.3 and Fluent 6.3. The geometries of the two impeller-tank configurations are created. They are meshed with approximately 500.000 tetrahedral elements: the size of the elements in the rotor zone and the stator zone are $1.5 \mathrm{~mm}$ and $3 \mathrm{~mm}$, respectively. The Sliding Meshes (SM) model is preferred to the Moving Reference Frame (MRF) model as it has been shown that it determines the dissipated power with more accuracy. As the tank is unbaffled, in order to take up the effect of the free surface deformation, the Volume Of Fluid (VOF) multiphase approach is selected. The standard $\mathrm{k}-\varepsilon$ approach is selected to model the turbulence. The tank is filled with $10 \mathrm{~cm}$ of methanol.

The first order-upwind discretization scheme is selected for the momentum, the turbulent kinetic energy and the turbulent dissipation range. The discretization scheme selected for the pressure and the volume fraction are the PRESTO! and the geo-reconstruct, respectively. The pressure-velocity coupling scheme recommended for this kind of simulation is the PISO.

In order to reach the convergence and conserve a courant number inferior to 0.25 , the time-step used is $5.10^{-5} \mathrm{sec}$.

For each simulation, the dissipated power is determined by calculating the total moment forces on the impeller shaft. 
Table 2: $\quad$ Density and viscosity of the reference Newtonian liquids used.

\begin{tabular}{cccc}
\hline Substance & $\begin{array}{c}\text { Temperature } \\
{ }^{\circ} \mathrm{C}\end{array}$ & $\begin{array}{c}\text { Density, } \rho \\
\mathrm{kg} / \mathrm{m}^{3}\end{array}$ & $\begin{array}{c}\text { Viscosity, } \mu \\
\mathrm{mPa} . \mathrm{s}\end{array}$ \\
\hline Glycerine - Water & 10 & 1230 & 1372 \\
Glycerine - Water & 15 & 1230 & 1015 \\
Glycerine - Water & 20 & 1230 & 751 \\
Glycerine - Water & 25 & 1220 & 556 \\
Glycerine - Water & 30 & 1220 & 411 \\
Glycerine - Water & 35 & 1220 & 304 \\
Glycerine - Water & 40 & 1220 & 225 \\
Glycerine - Water & 50 & 1210 & 123 \\
Glycerine - Water & 60 & 1210 & 67 \\
- Salt - Water & 5 & 1239 & 3.09 \\
Salt - Water & 10 & 1237 & 2.01 \\
Salt - Water & 15 & 1234 & 2.32 \\
Salt - Water & 20 & 1231 & 1.53 \\
Methanol & 20 & 780 & 0.55 \\
\hline
\end{tabular}

\section{Results and discussion}

The experimental results of the Power number as a function of the Reynolds number and the Froude number are obtained for the two impeller-tank configurations in the extended Reynolds number range. The results indicate that there is not a significant influence of the Froude number on the Power number. Therefore, in the intermediate range, the relation links the Power number to the Reynolds number only. The least square optimisation in the intermediate range leads to two relations. The first one is valid for $\operatorname{Re}<500$, and the second one for $500<\operatorname{Re}<10^{4}-10^{5}$. The Power number in the turbulent range $\left(\operatorname{Re}>10^{4}-10^{5}\right)$ is constant. The relations $\mathrm{N}_{\mathrm{p}}-\mathrm{Re}$ are reported in Tab.3.

Table 3: $\quad \mathrm{N}_{\mathrm{p}}-\mathrm{Re}-\mathrm{Fr}$ relation for the two impeller-tank configurations.

\begin{tabular}{llcc}
\hline Impeller & Range & $\mathrm{c}$ & $\mathrm{x}$ \\
\hline Anchor & Intermediate $(\mathrm{Re}<500)$ & 0.499 & -0.541 \\
& Intermediate $\left(500<\mathrm{Re}<10^{4}-10^{5}\right)$ & 83.811 & -1.369 \\
& Turbulent $\left(\mathrm{Re}>10^{4}-10^{5}\right)$ & 0.0025 & 0 \\
\hdashline$\ldots \ldots \ldots \ldots$ & 0.465 & -0.465 \\
4 -arms FBT & Intermediate $(\operatorname{Re}<500)$ & 58.343 & -1.275 \\
& Intermediate $\left(500<\operatorname{Re}<10^{4}-10^{5}\right)$ & 0.005 & 0 \\
\hline
\end{tabular}

The experimental results of the Power number as a function of the Reynolds number are presented in Fig.3 for the 4-arms Flat Blade Turbine. The curve adjusting on the experimental results related to the Anchor is also presented. It can be observed that the curves of both impeller-tank configurations in the intermediate range are quite similar. However, as it can also be seen in the 
literature [5], the Power number in the turbulent range is bigger for the 4-arms Flat Blade Turbine than for the Anchor.

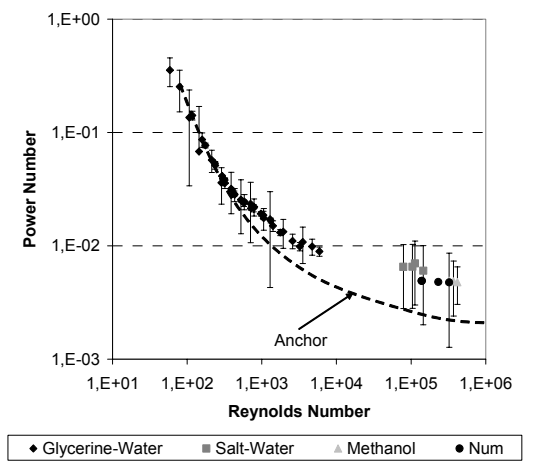

Figure 3: Power number as a function of the Reynolds number for the two impeller-tank configurations.

Fig.4 (left) presents the comparison of the Power number as a function of the Reynolds number in the turbulent range obtained both experimentally and numerically by CFD. Fig.4 (right) shows that at least 5 revolutions of the impeller are required to reach the numerical stationary conditions.
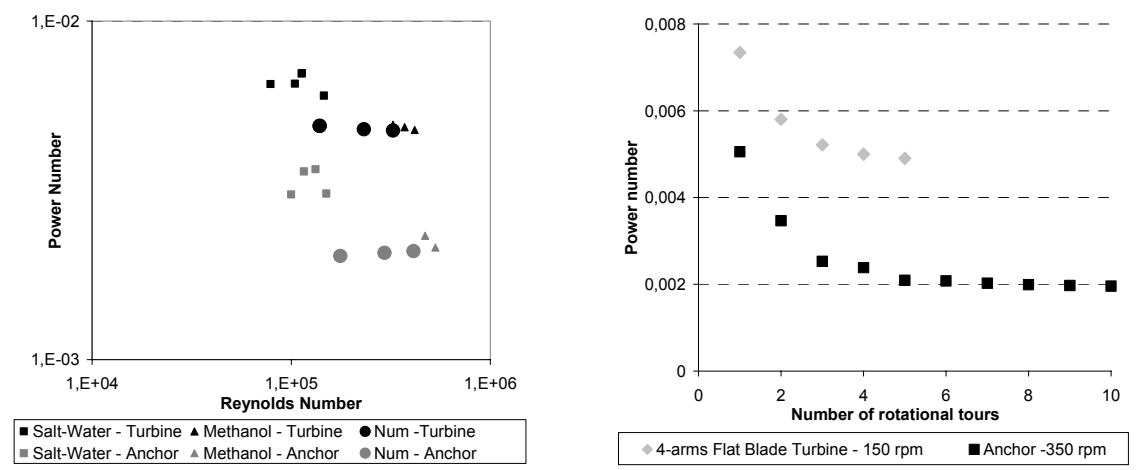

Figure 4: (Left) Comparison between the experimental and the numerical results of the Np vs. Re in the turbulent range. (Right) Numerical evolution of the Power number with the increase of the number of the rotational tours.

The Power numbers obtained are smaller that those generally found in the literature. Indeed, the important deformation of the free surface leads to a limitation of the dissipated power [5]. Moreover, with these impeller-tank configurations, the turbulent range is reduced. This characteristic is useful for the 
method proposed. Indeed, Eq.(5) can be used to determine the apparent viscosity of a suspension assuming that the relation between the Power number and the Reynolds number is a bijective one.

\section{Application}

The method is then applied for the determination of the evolution of the apparent viscosity of the crystal slurry during a crystallization process. The process chosen consists of the re-crystallization of the Etiracetam product (racemic compound (R-S product)): an intermediate product of the Levetiracetam (UCB Keppra). In this crystallization process, the drug can crystallize into two crystallographic forms, called morph I and morph II. The morph I is the stable crystallographic form below the transition temperature $\left(30^{\circ} \mathrm{C}\right)$ while the morph II is the stable crystallographic form beyond this temperature.

The reference pharmaceutical crude compound is initially dissolved $(0.6$ $\left.\mathrm{g}_{\text {product }} / \mathrm{g}_{\text {solution }}\right)$ in the methanol solvent at high temperature $\left(\sim 60^{\circ} \mathrm{C}\right)$ and stirred in a tank. The cooling of this solution $\left(15.5^{\circ} \mathrm{C} / \mathrm{h}\right.$, started at time $\left.\mathrm{t}=0\right)$ induces the crystallization of both morphs. Nevertheless, the non-desired crystallographic form (morph II) nucleation kinetics is dominant. The massive primary nucleation of morph II is observed at the so-called induction time $t=t_{1}$ by the first exothermic peak on the temperature curve (curve 1) in Fig.5. In order to obtain the pharmaceutical desired crystallographic form (morph I), the temperature is further lowered and the system is kept at a constant ripening temperature $\left(T_{\text {rip }}\right)$. At the so called latency time $t=t_{2}$, a polymorphic transition from the unstable morph II to the stable morph I is observed. The transition is characterized by a second exothermic peak on the temperature curve (curve 1) in Fig.5. This transition, mediated by the solvent, is controlled by the morph I nucleation.

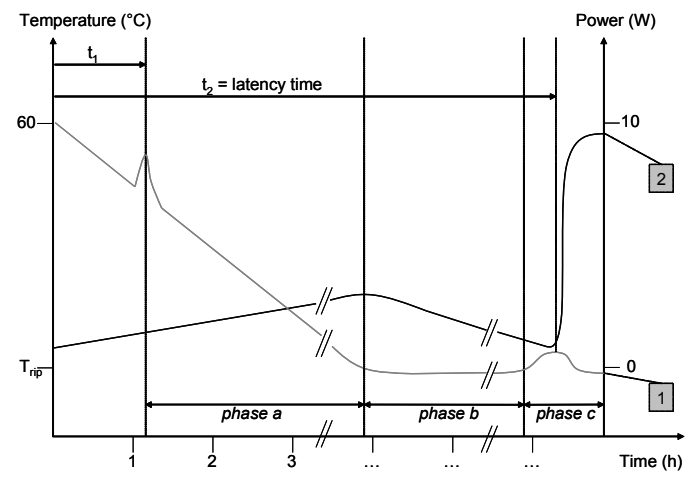

Figure 5: Evolution of the temperature (curve 1) and the power dissipated (curve 2) in the suspension during the polymorphic crystallization process. 
In order to determine the evolution of the apparent viscosity of the suspension during the crystallization process (curve 2 in Fig.5), it is realized in the impeller-tank configurations presented in Tab. $1(\mathrm{H}=10 \mathrm{~cm})$.

Several rotational speeds and ripening temperatures are investigated in order to discuss the evolution of the apparent viscosity of the suspension during the crystallization process as a function of the operational conditions. On the one hand, the influence of the mixing is investigated. For this purpose, two rotational speeds are investigated for both configurations ( $250 \mathrm{rpm}$ and $350 \mathrm{rpm}$ ) fixing the ripening temperature equal to $-2^{\circ} \mathrm{C}$. On the other hand, the influence of the ripening temperature is investigated. For this purpose, three temperatures are investigated $\left(-10^{\circ} \mathrm{C},-2^{\circ} \mathrm{C}\right.$ and $\left.10^{\circ} \mathrm{C}\right)$ fixing the agitation (Anchor $-250 \mathrm{rpm}$ ).

The power dissipated in the suspension during the crystallization process (curve 2 in Fig.5) is recorded for each experimental condition. The evolution of the apparent viscosity of the suspension is determined using the method proposed (Eq.(5)) assuming that the density of the suspension is constant $\left(960 \mathrm{~kg} / \mathrm{m}^{3}\right)$.

For each experiment, the morph II and morph I PSD, recorded at the end of the phase a and c, respectively, are off-line determined on dry powder with a MasterSizer. The average particle size $(\mathrm{D}[4,3])$, the median $(\mathrm{D}(\mathrm{v}, 0.5))$ and the span distribution $([\mathrm{D}(\mathrm{v}, 0.9)-\mathrm{D}(\mathrm{v}, 0.1)] / \mathrm{D}(\mathrm{v}, 0.5))$ are reported in this paper.

\subsection{Influence of the Particle Size Distribution of the crystals of morph I and morph II}

Fig.6 (left) presents the dissipated power measured during the crystallization process using the Anchor rotating at $350 \mathrm{rpm}$. The evolution of the apparent viscosity of the suspension, determined by the method, is also presented. The curves present a drastic change between the end of phase a and the end of phase c. In both cases, there are approximately $50 \%$ in mass of solid crystals in the suspension. Therefore, this change may be explained by the difference in the PSD and number of morph II and morph I crystals in suspension.

At the end of the phase a, the density and the size of the morph II solid particles are large and they quickly settle down after stopping the rotation of the impeller. In this case, the flow is in a intermediate state and the suspension is a Newtonian liquid. At the end of the phase c, the density and the size of the morph I solid particles are smaller than those of the morph II crystals (Fig.6 (right)) and the amount of solid suspended is more abundant, so that the particles do not settle down quickly. In this case, the flow is in a laminar state, and the suspension is a non-Newtonian liquid.

The analysis of both Fig.6 seems to indicate that, for a same crystal mass fraction in the suspension, the smaller the median is (and then the smaller and the more abundant the crystals are) and the bigger the span is, the bigger the apparent viscosity of the suspension is. 

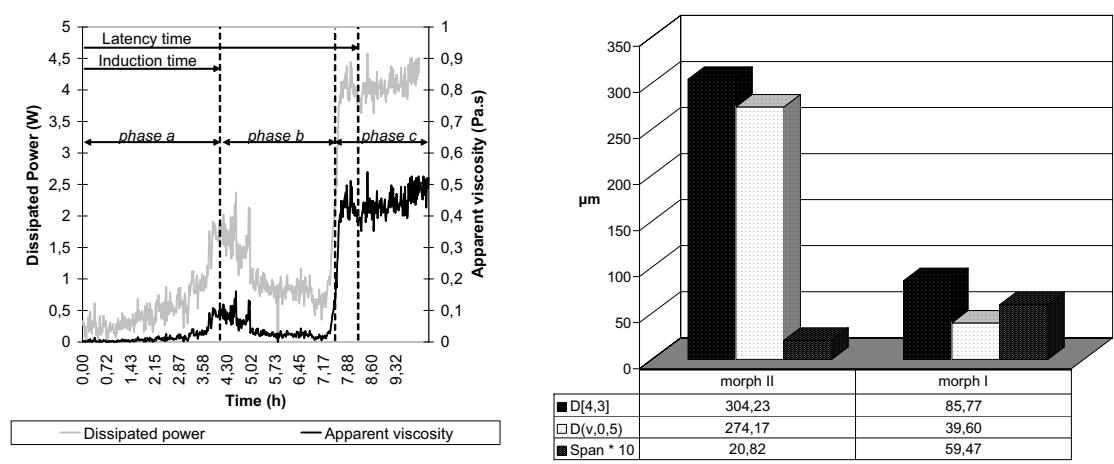

Figure 6: (Left) Evolution of the dissipated power and the apparent viscosity of the suspension (Anchor - 350rpm). (Right) Average morph II (end of phase a) and morph I (end of phase c) PSD.

\subsection{Influence of the mixing}

The influence of the mixing on the evolution of the apparent viscosity of the crystal slurry is investigated by 4 experimental conditions. Both impeller-tank configurations (Tab.1) are used with two rotational speeds $(250 \mathrm{rpm}$ and $350 \mathrm{rpm})$.

Fig.7 (left) presents the apparent viscosity at the end of the phase a (morph II) and at the end of the phase $\mathrm{c}$ (morph I) for the four mixing experimental conditions. For each of them, the total mass of morph II crystals and morph I crystals at the end of the phase a and c, respectively, is the same. Moreover, they are approximately $50 \%$ in mass of crystals in the suspension. It can be observed that the apparent viscosity at the end of each phase depends on the impeller-tank configuration but is quite independent on the rotational speed investigated. These results can be discussed with the results of the morph I PSD (Fig.7 (right)) for the four experimental conditions.

It can be seen that, for the two impeller-tank configurations, D[4,3] and $\mathrm{D}(\mathrm{v}, 0.5)$ are reduced when the rotational speed is increased while the span does not present a significant change. Fig.7 also indicate that, for a given rotational speed, the morph I PSD related to the Turbine is more extended (bigger span) than those related to the Anchor while they present a same median. This induces a bigger heterogeneity of the particle size of the morph I crystals in the suspension. As previously, this may explain the difference in the apparent viscosity of the suspension obtained when agitating with the Anchor or the Turbine. For a same crystal mass fraction and a same median, the bigger the span is, the bigger the apparent viscosity of the suspension is. 

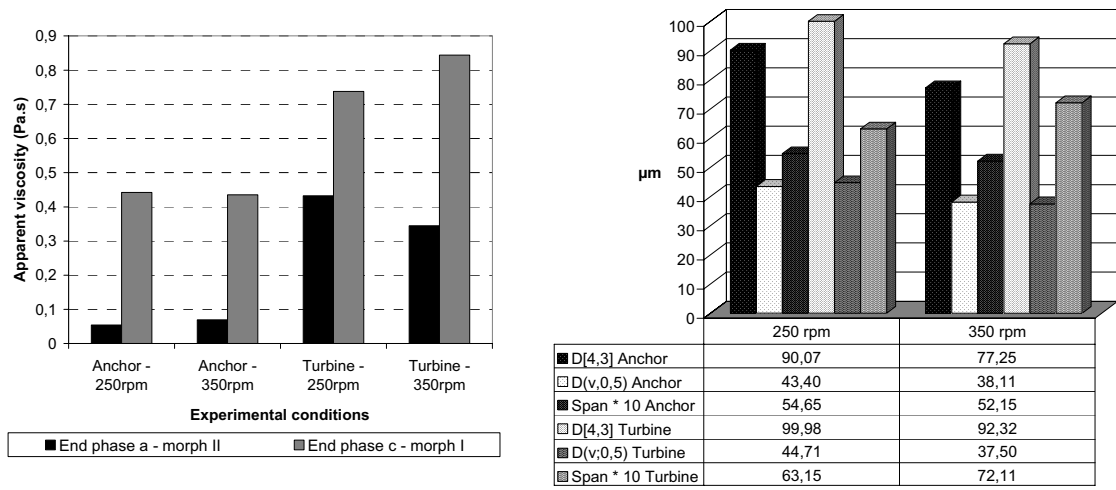

Figure 7: For the four experimental conditions investigated. (Left) Apparent viscosity at the end of phase a and phase c. (Right) Morph I PSD.

\subsection{Influence of the ripening temperature}

Three ripening temperatures are investigated in order to study their influence on the time evolution of the apparent viscosity of the crystal slurry.

Fig.8 (left) presents the dissipated power in the suspension at the end of the phase $\mathrm{a}$ and at the end of the phase $\mathrm{c}$ for the three ripening temperatures investigated. Fig. 8 (right) present the morph I PSD at the end of the phase c.
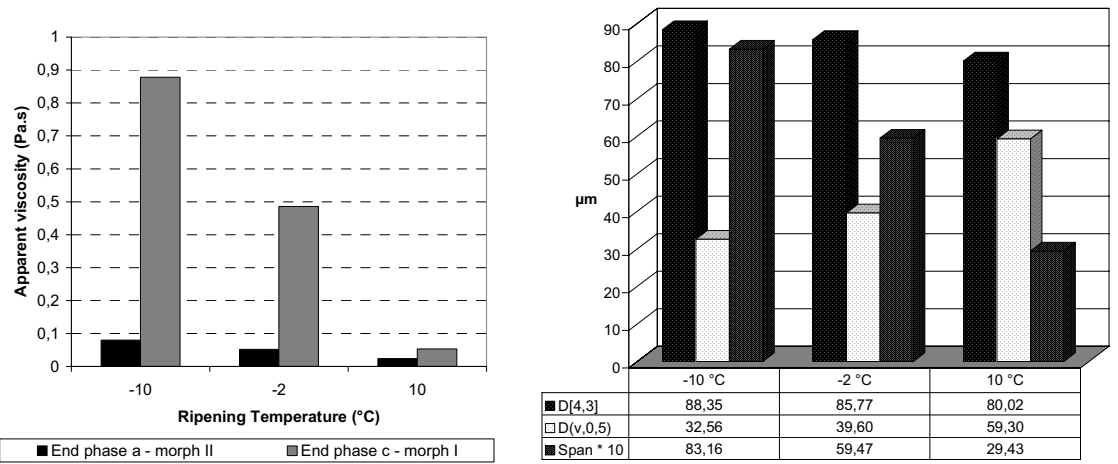

Figure 8: For the three experimental conditions investigated. (Left) Apparent viscosity at the end of phase a and phase c. (Right) Morph I PSD.

As previously, these Fig. 8 allow one to conclude that the bigger the crystals are and the smaller the span is, the smaller the apparent viscosity of the suspension is. Moreover, when reducing the ripening temperature from $10^{\circ} \mathrm{C}$ to $-10^{\circ} \mathrm{C}$, as the solubility decreases, the mass and number of the crystals in suspension increase leading to an augmentation of the apparent viscosity . 


\section{Conclusion}

This paper presents an experimental method to follow the apparent viscosity of a crystal suspension during a crystallization process, based on the Metzner and Otto concept. The first part of this paper focuses on the experimental and numerical (by CFD) determination of the $\mathrm{N}_{\mathrm{p}}-\mathrm{Re}-\mathrm{Fr}$ relation for Newtonian liquid for two impeller-tank configurations (unbaffled tank, Anchor or 4-arms Flat Blade Turbine). These one are chosen in order to obtain a bijective relation between the Power number and the Reynolds number in an extended Reynolds range. The method proposed is then described and applied to on reference pharmaceutical crystallization process. In order to analyse the influence of the solid phase characteristics on the apparent viscosity of the suspension, several operational conditions (mixing and temperature) are investigated. The paper concludes on the influence of the Particle Size Distribution of the crystals on the apparent viscosity of the suspension. For a given crystal mass fraction, the bigger the crystals are - and then the less abundant they are - and/or the smaller the span is, the smaller the apparent viscosity of the suspension is.

\section{Acknowledgements}

Christelle Herman acknowledges the Fonds National de la Recherche Scientifique (FNRS), UCB Pharma and the Hubert Currien (Tournesol) partnership for their financial support.

\section{References}

[1] Mullin, J.W., Crystallization, Elsevier, Oxford, UK, 2001

[2] Roscoe, R., The viscosity of suspension of rigid spheres, J. Appl. Phys., 3, pp. 267-269, 1952

[3] Snabre, P. and Mills, P., Rheology of concentrated suspensions of viscoelastic particles, Colloïd and Surfaces A., Physicochemical and engineering aspects, 152(1-2), pp. 79-88, 1999

[4] Metzner, A.B. and Otto, R.E., Agitation of Non-Newtonian Fluids, AIChE Journal, 3(1), pp. 3-10, 1957

[5] Nagata, S., Mixing: Principles and applications, A. Halsted press book, Tokyo, Japan, 1975 